\section{OP0320 SUCCESSFUL EVALUATION OF A PREDEFINED SET OF ANATOMIC SITES IN THE PELVIS OF PATIENTS WITH POLYMYALGIA RHEUMATICA SHOWING EXTRACAPSULAR INFLAMMATION AS VISUALIZED BY CONTRAST ENHANCED MAGNETIC RESONANCE IMAGING:}

Martin Fruth ${ }^{1}$, J Kozik ${ }^{1}$, P Martin-Seidel ${ }^{1}$, Annika Seggewiss ${ }^{1}$, Xenofon Baraliakos ${ }^{2,3}$, Juergen Braun ${ }^{2,3} .{ }^{1}$ Radiology, Herne, Germany; ${ }^{2}$ Rheumazenrum Ruhrgebiet, Herne, Germany; ${ }^{3}$ Ruhr-University, Bochum, Germany

Background: The diagnosis of polymyalgia rheumatica (PMR) is based on a thorough clinical evaluation of the patient - including exclusion of other diseases, since there is no decisive diagnostic test. A characteristic pattern of extracapsular inflammation in the pelvis of patients with PMR as assessed by contrast enhanced magnetic resonance imaging (MRI) has been recently described (1)

Objectives: To evaluate the performance of a predefined set of anatomic sites in the pelvis of patients with PMR vs. controls.

Methods: A total of 120 pelvic MRI scans of patients who had presented to our tertiary center with pelvic girdle pain in the last 3 years, including 40 patients with an expert rheumatologist diagnosis of PMR and 80 controls with other reasons of pelvic pain was evaluated by 3 radiologists blinded to clinical diagnosis and patient demographics. The experts scored the presence or absence of contrast enhancement at 19 predefined tendinous and capsular pelvic structures. Different patterns of involvement were compared and statistically evaluated by ROC analysis. Kappa statistics were applied to calculate inter- and intrareader agreement. Results: Mostly bilateral peritendinitis and capsulitis including uncommon sites such as the proximal origins of the muscles rectus femoris and adductor longus were found almost exclusively and, thus, typically in PMR patients: the difference in the mean number of sites showing contrast enhancement was significantly different with $13.4 \pm 2.7$ for PMR vs $4.0 \pm 2.3$ for controls. A cut-off of $\geq 10$ inflamed sites discriminated very well between the groups resulting in a sensitivity and specificity of $95.8 \%$ and $97.1 \%$, respectively. Just concentrating on the most frequently involved anatomic sites bilateral inflammation of proximal M. rectus femoris or adductor longus tendons together with at least 3 other bilaterally inflamed sites performed even better with a sensitivity and specificity of $100 \%$ and $97.5 \%$, respectively.

Conclusion: This study strongly confirms that the previously described pattern of extracapsular pelvic inflammation as assessed by contrast enhanced MRI is very typical for patients with PMR. In addition, the high sensitivity and specificity of the set of anatomic sites evaluated suggest their definite potential for use as a confirmatory diagnostic test.

\section{REFERENCE:}

[1] Fruth M, Buehring B, Baraliakos X, Braun J. Use of contrast-enhanced magnetic resonance imaging of the pelvis to describe changes at different anatomic sites which are potentially specific for polymyalgia rheumatica. Clin Exp Rheumatol. 2018; 36 Suppl 114(5):86-95.

Disclosure of Interests: Martin Fruth: None declared, J Kozik: None declared, P Martin-Seidel: None declared, Annika Seggewiss: None declared, Xenofon Baraliakos Grant/research support from: AbbVie, Boehringer Ingelheim, Bristol-Myers Squibb, Celgene, Centocor, Chugai, Janssen, MSD, Novartis, Pfizer Inc, Roche and UCB, Grant/research support from: AbbVie, Pfizer, Merck Sharp \& Dohme, UCB Pharma, Novartis, Consultant for: AbbVie, Bristol-Myers Squibb, Boehringer Ingelheim, Celgene, Chugai, Janssen Biologics, Novartis, Pfizer, UCB Pharma, Galapagos, Speakers bureau: AbbVie, Chugai, Janssen, Novartis, Pfizer, UCB Pharma, Juergen Braun Shareholder of: AbbVie, BMS, Celgene, Chugai, Merck, Novartis, Pfizer, UCB, Grant/research support from: AbbVie, BMS, Celgene, Chugai, Merck, Novartis, Pfizer, UCB, Grant/research support from: Abbott, Bristol Myers Squibb, Celgene, Celltrion, Chugai, Johnson \& Johnson, MSD, Novartis, Pfizer, Roche, UCB Pharma, Grant/research support from: AbbVie, BMS, Celgene, Chugai, Merck, Novartis, Pfizer, UCB, Grant/research support from: Abbvie (Abbott), Amgen, Baxter, Biogen, BMS, Boehringer, Celgene, Celltrion, Centocor, Chugai, Hexal, Janssen, Lilly, Medac, MSD (Schering-Plough), Mylan, Mundipharma, Novartis, Pfizer (Wyeth, Hospira), Roche, Sanofi-Aventis and UCB, Consultant for: Abbvie (Abbott), Amgen, Baxter, Biogen, BMS, Boehringer, Celgene, Celltrion, Centocor, Chugai, Hexal, Janssen, Lilly, Medac, MSD (ScheringPlough), Mylan, Mundipharma, Novartis, Pfizer (Wyeth, Hospira), Roche, SanofiAventis and UCB, Consultant for: AbbVie, BMS, Celgene, Chugai, Merck, Novartis, Pfizer, UCB, Consultant for: Abbott, Bristol Myers Squibb, Celgene, Celltrion, Chugai, Johnson \& Johnson, MSD, Novartis, Pfizer, Roche, UCB Pharma, Speakers bureau: AbbVie, BMS, Celgene, Chugai, Merck, Novartis, Pfizer, UCB, Speakers bureau: Abbvie (Abbott), Amgen, Baxter, Biogen, BMS, Boehringer, Celgene, Celltrion, Centocor, Chugai, Hexal, Janssen, Lilly, Medac, MSD (ScheringPlough), Mylan, Mundipharma, Novartis, Pfizer (Wyeth, Hospira), Roche, SanofiAventis and UCB, Speakers bureau: AbbVie, BMS, Celgene, Chugai, Merck, Novartis, Pfizer, UCB
DOI: 10.1136/annrheumdis-2019-eular.5332

Mitchell Silva, Nele Caeyers. ReumaNet, zaventem, Belgium

Background: Every two years, ReumaNet organises a physical conference for patients addressing various topics of rheumatic and musculoskeletal diseases (RMD). Traditionally, about 200 individuals attend this event. For the last edition however, ReumaNet swapped the physical event with a virtual one. This allowed us to reach far more people, who could attend the 'conference' online whenever they wanted, over a period of four weeks.

Objectives: The objective was to assess the impact, reach and patient experience of such a virtual event.

Methods: In this virtual event, more than twenty presentations were pre-recorded and put into an online system. Virtual booths were offered to partner organisations to offer educational material in pdf's or in video format.

The board of ReumaNet set up an interesting program, covering the following aspects:

- Medical evolution in RMD's: explanation about new treatments in various indications, information on new medication

- Living with an RMD: information about the psychological, vocational, social and physical aspects of having an RMD

- The future of healthcare: information on the changing technology in healthcare and sustainability of the healthcare system

- Testimonials: video testimonials of patient advocates

All information was pre-recorded and integrated into an online portal. The lectures showed both speaker and the slide set, moving along with the presentation. Registration was free and anonymous. Only email was required in order to validate the registration. Visitors also had the option to answer surveys on various topics and score every video presentation. All material could be downloaded (PowerPoint presentations, brochures, videos).

This virtual symposium was accessible from mid-September till mid-October, covering four weeks around World Arthritis Day. People could come and go, and log in at any other point in time from different devices, so it was not necessary to view all at once.

Results: The results were very promising, having over 1.300 registrations (compared to 200 registrations at a physical event). The social media reach was over 140.000 and the event also increased the visibility of the Facebook page of ReumaNet. On the platform itself there were over 5000 video views and over 3000 vis its of the virtual booths of partner organisations. General satisfaction rates were high: $96 \%$ of the visitors indicated they were most likely to visit a similar event in the future. Over $80 \%$ gave the event a +4 star rating out of 5 .

Conclusion: This event was considered to be very successful. The results showed that this virtual event exceeded our expectations and had an impact on the visibility of our organisation via social media. This type of event has been expanded towards sister organisations in rheumatology, such as the Belgian organisation for healthcare professionals and can serve as an innovative way for other European patient organisations to attract new profiles and increase awareness of RMDs among the broad public.

Disclosure of Interests: Mitchell Silva Consultant for: For Pfizer, MSD, Janssen, Merck, BMS, Sanofi, Novartis, AstraZeneca, Speakers bureau: For Pfizer, MSD, Janssen, Merck, BMS, Sanofi, Novartis, AstraZeneca, Nele Caeyers: None declared DOI: 10.1136/annrheumdis-2019-eular.3281

\section{OP0322-PARE GRASSROOTS CAMPAIGN FOR DUTCH RMD- FRIENDLY MUNICIPALITIES}

Margot Feith, Nique Lopuhaä, Sija De Jong. Dutch Arthritis Society, Patient Advocacy, Amsterdam, Netherlands

Background: Following the decentralisation of healthcare in 2014, Dutch municipalities were assigned a key role in providing healthcare support to local residents. ReumaNederland used the 2018 council elections to start a campaign to raise awareness for RMD-friendly council policies.

Objectives: The campaign aimed to raise awareness for suitable RMD healthcare policies across municipalities. 
Methods: Patient consultation - We asked people with RMD to complete an online survey about council policies. The survey asked whether, and how, these policies restricted them, in addition to what could be done to address and solve these restrictions. The questions focused on healthcare, support and the provision of medical aids. After identifying the three greatest challenges for residents, the Dutch Arthritis Society (DAS) focused on three main recommendations, which were sent via letter to local politicians. This letter included a clear call to action for better local arthritis care.

Online tool - We also created an online tool that linked the letter to the email addresses of all local party leaders in the 380 Dutch municipalities. The online tool enabled local residents to simply forward the letter to the party leaders in their council. The tool also allowed residents to include their personal experiences. Results: Patient consultation: More than 2,200 people with RMD completed the survey. The three greatest points of improvement included:

- Sports and leisure facilities for people with RMD - $80 \%$ of all respondents were interested in sport facilities that suit their needs. However, $75 \%$ were unsure if there were any available in their council.

- Better communication and information - $40 \%$ of all respondents indicated that it was unclear where and how they could apply for care with their council. Respondents also indicated that councils lacked expertise regarding the impact of $\mathrm{RMD}$.

- A threshold-free and accessible RMD-friendly environment - Municipalities must consider RMD-friendly access to roads, public transport and public buildings.

Online tool: A total of 10,000 emails were sent to party leaders, reaching over half of all municipalities. The majority of feedback from party leaders was positive. Some residents were invited to introduce themselves, attend a meeting or take a seat on a client advisory board. RMD-friendly policies are also now featured on the political agenda of many local councils.

Conclusion: This campaign has put RMD-friendly policies on the council agenda in many municipalities. Clearly, using the momentum of local political developments is an effective tool to create attention. It is also a way to build the network and voice of the movement. Following the campaign, it has become easier for us to speak to the councillors responsible for healthcare and to address the issues raised by people with RMD. For instance, in the autumn of 2018, local RMD patient associations met with the council for the first time. The online tool has allowed for nation-wide participation in this campaign.

Disclosure of Interests: None declared

DOI: 10.1136/annrheumdis-2019-eular.4573

FRIDAY, 14 JUNE 2019

\section{Overdiagnosis and overtreatment in inflammatory} arthritis

\section{OP0323 USEFULNESS OF UNIVERSAL ANTINUCLEAR ANTIBODIES TESTING IN EARLY ARTHRITIS REFERRALS}

Mariana Luis, Luisa Brites, Ana Rita Prata, Tânia Santiago, José Antonio P. Da Silva, Catia Duarte. Centro Hospitalar e Universitário de Coimbra, Rheumatology, Coimbra, Portugal

Background: The CHUC Early Arthritis Clinic, founded in 2011, intends to provide a prompt response to patients with recent onset of symptoms suggestive of an inflammatory rheumatic disease (IRD). By research protocol, all patients are screened at baseline for the presence of antinuclear antibodies (ANA). An analysis was performed to evaluate the usefulness of universal ANA testing in these patients.

Objectives: To evaluate the prevalence and clinical correlates of ANA positivity in early arthritis referrals.

Methods: A retrospective study of consecutive patients referred to the Early Arthritis Clinic between 2011 and 2018 was conducted. Referral is based on the fulfillment of specific criteria: presence of arthritis or clinically suspected arthralgia beginning in the previous 12 months plus suggestive laboratorial abnormalities (rheumatoid factor, C-reactive protein or erythrocyte sedimentation rate). ANA titer (positive $=\geq 1: 160$ ) and cellular staining patterns were assessed by indirect immunofluorescence (Hep-2 cells). Positive (PPV) and negative predictive values (NPV) of an ANA positive test for the diagnosis of an IRD or an ANA-related rheumatic disease (ARD) were determined, along with PPV for the other referral criteria.

Results: 207 patients were included in the analysis (64.3\% female, aged $53.9 \pm$ 18.2 years). The diagnosis of an IRD was confirmed by the rheumatologist in
$61.4 \%$ of cases, including $11.8 \%$ cases of ARD. The most prevalent diagnosis was rheumatoid arthritis $(21.7 \%)$, followed by unclassified arthritis $(8.7 \%)$, psoriatic arthritis, osteoarthritis and fibromyalgia (6.8\% each). The prevalence of ANA positivity in our cohort was $64.2 \%$, most frequently in low titration $(1: 160$ in $33.8 \%$, $1: 320$ in $19.3 \%, 1: 640$ in $8.7 \%$ and $1: 1280$ in $2.4 \%$ ) and with a dense fine speckled pattern (45.1\%). ANA-positive patients were older $(53.7 \pm 17.9$ versus $47.9 \pm$ $18.5, p=0.05)$, more likely to have an IRD $(72.9 \%$ versus $40.5 \%, p<0.001)$ but not an ARD $(9.0 \%$ versus $4.1 \%, p=0.186)$. PPV for ARD was $9.0 \%$ for the $1: 160$ cutoff titration and $15.6 \%$ for the $1: 320$. Squeeze test (PPV 13.5\%), erythrocyte sedimentation rate (PPV 9.9\%) and rheumatoid factor (PPV 20.0\%), as referral criteria, all performed better at predicting an ARD, compared to an ANA positive testing.

Conclusion: Early Arthritis Clinic referred cohort has a high prevalence of IRD but a low prevalence of ARD which explains the poor predictive value of ANA in this setting, especially when considering lower titters and when compared with specific referral criteria. Thus, universal ANA testing in Early Arthritis Clinic refer rals seems unjustified, given its costs and added value. Studies designed to optimize the use of ANA in this context are warranted.

Disclosure of Interests: None declared

DOI: 10.1136/annrheumdis-2019-eular.5053

\section{FRIDAY, 14 JUNE 2019}

\section{Cannabis for arthritis: Hype or hope?}

\section{OP0324 \\ CANNABIDIOL ELEVATES INTRACELLULAR CALCIUM AND INDUCES APOPTOSIS IN HUMAN ARTICULAR CHONDROCYTES}

Martina Winklmayr ${ }^{1,2,3}$, Martin Gaisberger ${ }^{1,2,3}$, Julia Fuchs ${ }^{1,2,3}$, Michael KittlI ${ }^{1}$, Markus Ritter ${ }^{1,2,3}$, Martin Jakab'. ${ }^{1}$ Paracelsus Medical University, Institute of Physiology and Pathophysiology, Salzburg, Austria; ${ }^{2}$ Ludwig Boltzmann Cluster for Arthritis and Rehabilitation, Wien, Austria; ${ }^{3}$ Gastein Research Institute, Salzburg, Austria

Background: Osteoarthritis $(\mathrm{OA})$ is a major public health problem among the increasing aged and obese population, therefore development and investigation of new therapeutics is a major focus of OA research. Endocannabinoids (ECs), cannabinoids derived from the Cannabis sativa plant and synthetic cannabinoids have been attributed anti-inflammatory, antitumorigenic, analgesic and psychoactive effects. Over recent years increasing interest in the EC system as a target for therapeutic treatment of joint diseases has emerged [1].

Objectives: Cannabidiol (CBD) is the most abundant non psychoactive compound of Cannabis sativa extracts and has been shown to have anti-arthritic potency in animal models [2, 3]. In the present study we investigated the effects of $\mathrm{CBD}$ on the cell viability and $\mathrm{Ca}^{2+}$ homeostasis in human articula chondrocytes.

Methods: Cell viability, discrimination of intact, apoptotic and necrotic cells and caspase 3/7 activity were determined by Resazurin assays, Annexin-V/7-AAD staining followed by flow cytometry and caspase-Glo 3/7 assay respectively. Intracellular $\mathrm{Ca}^{2+}$ was monitored by time-lapse fluorescence imaging. The perforated whole-cell patch clamp technique was used for measuring the cell membrane potential. Western blot analysis was performed for the quantification of Erk1/2 phosphorylation.

Results: C28/i2 and human primary chondrocytes showed a significantly reduced viability with an apoptosis maximum at $10 \mu \mathrm{M} \mathrm{CBD}$ after treatment with rising amounts of CBD. This apoptotic effect was accompanied by an increase of caspase $3 / 7$ activity. Flow cytometry analysis of Annexin-V/7-AAD stained cells revealed a decline of intact cells and a significant dose dependent increase of the early apoptotic cell population after treatment with CBD.

CBD significantly elevated intracellular $\mathrm{Ca}^{2+}{ }_{i}$ accompanied by a depolarization of the cell membrane. This increase of $\mathrm{Ca}^{2+}{ }_{i}$ was abrogated, when $\mathrm{Ca}^{2+}$ was omitted from the bath solution indicating an influx of extracellular $\mathrm{Ca}^{2+}$ rather than depletion of internal stores. Several blocking substances were tested to identify the channel/receptor responsible for this $\mathrm{Ca}^{2+}$ influx. Cannabinoid receptor1 (CB1) antagonist AM251 significantly inhibited the $\mathrm{Ca}^{2+}$ influx trig gered by CBD. Moreover, preincubation of chondrocytes with AM251 significantly reduced the toxic effects of $C B D$. Looking for mediators of the apoptotic CBD effect downstream of the $\mathrm{CB} 1$ receptor enhanced Erk1/2 phosphorylation could be detected. However this Erk1/2 activation proved to be unaffected by CB1 receptor blockage.

Conclusion: Micromolar concentrations CBD induce apoptosis in human articular chondrocytes. CBD also triggers an influx of extracellular $\mathrm{Ca}^{2+}$ and potentiates Erk1/2 phosphorylation. The apoptotic effects are at least partially 